\title{
WHOLE BODY VIBRATION TRAINING IMPROVES MUSCULAR POWER IN A RECREATIONALLY ACTIVE POPULATION
}

\author{
Adam Hawkey ${ }^{1}$ \\ ${ }^{1}$ Research Centre for Sport, Exercise and Performance, University of Wolverhampton, \\ United Kingdom
}

doi: 10.5550/sgia.120801.en.116H

ORIGINAL SCIENTIFIC PAPER

\begin{abstract}
SUMMARY
Whole body vibration training has been shown to improve athletic performance. However, the majority of studies have utilised relatively intensive training programmes. The current study assessed the effect of a 6-week vibration training programme on a recreationally active population. Following institutional ethics approval, 22 healthy, recreationally active male individuals were recruited and randomly assigned into either a vibration group $(n=11)$ or a control group $(n=11)$. The vibration group undertook 6-weeks training, conducted once-a-week, utilising 3 different squatting exercises $\left(120^{\circ}\right.$ and $90^{\circ}$ static squats, and $120^{\circ}-90^{\circ}$ dynamic squat) on a NEMES Bosco vibration platform: Week 1 (3 x $60 \mathrm{~s}, 35 \mathrm{~Hz} / 2 \mathrm{~mm})$; Week 2 (3 x 70s, $35 \mathrm{~Hz} / 2 \mathrm{~mm})$; Week 3 (3 x 60s, $40 \mathrm{~Hz} / 2 \mathrm{~mm})$; Week 4 (3 x 70s, $40 \mathrm{~Hz} / 2 \mathrm{~mm})$; Week 5 ( 3 x 60s, $45 \mathrm{~Hz} / 2 \mathrm{~mm})$; Week 6 ( $3 \times \mathrm{x}$. lowed the same training programme with sham $(0 \mathrm{~Hz} / 0 \mathrm{~mm})$ vibration. Prior to, and on completion of, the study all participants performed 3 maximal countermovement jumps to assess muscular power. A 2-way ANOVA with repeated measures, with one between-subject factor "group" (experimental vs. control) and one within-subject factor "time" (pre vs. post intervention), indicated that there was a significant time $\mathrm{x}$ group interaction $(\phi<.0001)$ for countermovement jump performance between the intervention (pre- $.43 \pm .08 \mathrm{~m}$ to post- $.49 \mathrm{~m} \pm .08$ ) and control group (pre- $.43 \pm .07 \mathrm{~m}$ to post- .41 $\pm .08 \mathrm{~m}$ ). Results suggest that 6 -week vibration training conducted once-a-week is sufficient to elicit significant increases in jumping performance in a recreationally active population.
\end{abstract}

Key Words: jumping, performance, squatting, recreationally active, strength, power.

\section{INTRODUCTION}

Vibration can be described as a mechanical stimulus characterised by a recurring oscillatory motion back and forth over the same pattern. We experience vibration throughout our daily lives when driving a car or operating machinery with motorised parts and exposure is traditionally associated with negative effects on the human body. However, whole body vibration (WBV) training also referred to as vibration training (VT) or vibration exercise (VE) has been developed as a relatively recent advance in health and fitness. The most common method of receiving vibration, as a specific training modality, is through the use of a vibration platform, also known as a vibration plate (Figure 1). These platforms operate in mo- vements, which can be classified as either linear (vertically only), oscillating (moving vertically on alternate sides in a seesaw-like manner) or tri-planar (moving through all three axis), with the intensity of the training being manipulated by altering the frequency and/or amplitude of oscillations (Hawkey, 2012). The frequency of the vibration stimulus simply quantifies the number of impulses or oscillations $(\mathrm{Hz})$ delivered every second (the repetition rate of the cycles), while the amplitude corresponds to the extent of the vertical displacement from the centre point of movement or its equilibrium position (Riley \& Sturges, 1996). The acceleration of the movement is measured in g's, or magnitudes of gravity (where $1 \mathrm{~g}$ is the acceleration due to the earth's gravitational field or 9.81 $\mathrm{m} \cdot \mathrm{s}^{-2}$ ). The acceleration that a body experiences whi- 
le on a vibration platform can be estimated using the equation shown in Figure 2. (Where a is the acceleration experienced expressed as the equivalent of the acceleration of earth's gravity $\left[9.81 \mathrm{~m} . \mathrm{s}^{-2}\right]$; $\mathrm{A}$ is the amplitude of the vibration; and $\mathrm{f}$ is the frequency of the vibration). For this formula to work effectively it is vital to reduce each component to mass, length and time, so that force $=$ mass $\mathrm{x}$ length $\mathrm{x} \operatorname{time}^{-2}($ Hawkey, 2012).

Although the exact mechanisms are not yet fully understood, it is thought that WBV elicits changes through physiological adaptations to accommodate vibratory waves (Cardinale \& Bosco, 2003), via a process termed the tonic vibration reflex (TVR; Nordlund \& Thorstensson, 2007); originally proposed by Eklund and Hagbarth (1966) following research directly exposing tendons to vibration. It is believed that stimulation of neuromuscular pathways and muscle spindles (Ia. afferent) excites the motor-neurons, causing contraction of homonymous motor units (Luo, McNamara, \& Moran, 2005), which, according to Mester, Kleinoder, and Yue (2006), means that TVR can improve maximum voluntary contraction of muscles. However, the connection between WBV and the TVR has not been fully accepted, with others proposing alternative mechanisms of increased muscle temperature and blood flow (Issurin \& Tenenbaum, 1999), change of perception by vibration (Liebermann \& Issurin, 1997), increased hormone secretion (Cardinale \& Bosco, 2003), and motor-unit synchronization and the recruitment of previously inactive motor-units (Issurin \& Tenenbaum, 1999). Research has shown that performing traditional weight training on a vibration platform produces significantly greater improvements in maximal strength and explosive power compared to traditional weight training (Ronnestad, 2004), while increases in the rate of force development and a reduction in electromechanical delay, both beneficial for explosive muscular activation, have also been observed (Hong, Kipp, Maddalozzo, \& Hoffman, 2010).

Vibration training has therefore been utilised for a variety of purposes in both the medical and sporting arenas. Improvements in bone health of post-menopausal women (Rubin et al., 2004), balance, gait, and quality of life ratings of elderly patients (Bruyere et al., 2005), and the reduction of joint swelling in rheumatoid arthritis patients (Kumari, Wyon, Hawkey, \& Metsios, 2011) have all been observed, while the jumping performance of semi-professional football players (Hawkey, Evans, \& Nevill, 2012), professional football goalkeepers (Hawkey, Morrison, Williams, \& Nevill, 2009a), and athletes' sprinting, jump height and explosive strength endurance (Paradisis \& $\mathrm{Za}$ charogiannis, 2007) have all been enhanced by WBV programmes. However, not all research has reported such improvements; one study conducted on basketball players reported no changes in jumping performance following WBV exposure (Hawkey, Lau, \& Nevill, 2009b). The reasons for this disparity in findings could be multi-factorial: differing protocols, testing equipment, and the performance level or experience of the participants. While the majority of previous research has reported improvements in jumping performance in these trained populations, those conducted on sedentary or recreationally active individuals are limited. Torvinen, Kannu, and Sievanen (2002) found an 8.5\% improvement following a 4-month intervention in untrained individuals. However, no placebo group was utilised making it difficult to ascertain if the improvements resulted from the exercises that were performed on the platform or from the WBV intervention. Delecluse, Roelants, and Verschueren (2003), did include a control group and reported a nearly $17 \%$ improvement in knee extensor strength and an almost $8 \%$ increase in the jumping performance of sedentary individuals who adhered to a WBV training programme 3-times-a-week over a 12 -week period. With the popularity and availability of vibration platforms in fitness centres and gymnasia around the world reported to be increasing (Fischbach, 2007), there is a need to add further research to the limited amount of data available on the effects of WBV on untrained and recreationally active populations. Therefore, the aim of the current study was to examine the effects of a relatively short duration, low intensity, WBV training programme, on the jumping performance of a recreationally active population.

\section{METHODS}

Following institutional ethics approval, and the completion of informed consent forms and medical questionnaires, 22 healthy, recreationally active, male undergraduate sports students (age mean 28, $\mathrm{s}=10$ years; height mean 1.77, s $=.09 \mathrm{~m}$; mass mean $77, \mathrm{~s}$ $=13 \mathrm{~kg}$,) were randomised into either a WBV or control group. The vibration intervention consisted of 6-week WBV training, utilising three different squatting exercises (static half squat at $120 \mathrm{o}$, static deep squat at $90^{\circ}$ and a dynamic squat ranging from $120^{\circ}$ to $90^{\circ}$; monitored using a goniometer), conducted once a week, on a NEMES Bosco vibration platform (Figure 1). Prior to training and testing, both vibration and control groups completed a warm up, 


\section{FIRURE 1}

An athlete trains on the NEMES Bosco Vibration Platform (C) Adam Hawkey)

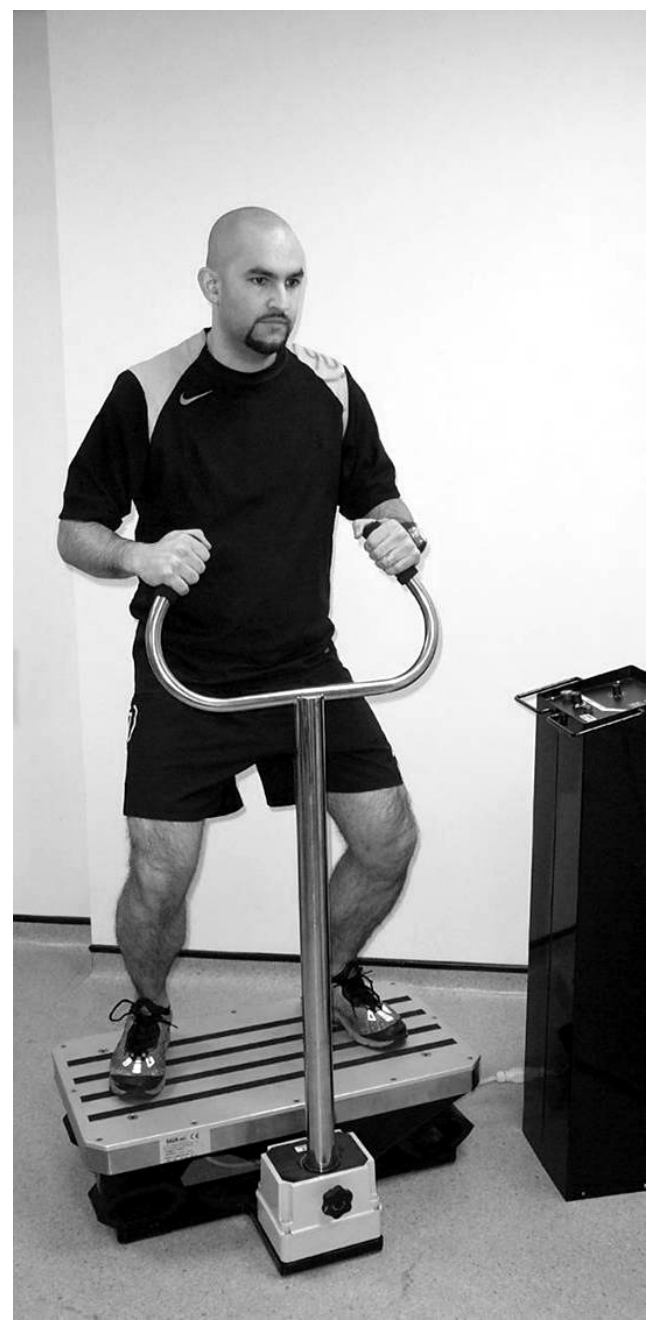

which consisted of 5 minutes on a Monark Cycle Ergometer with heart rate between 120-140 BPM in accordance with the American College of Sports Medicine (ACSM) who recommend a minimum of five to ten minutes of low- to moderate-level activity in order to increase muscle temperature (ACSM, 2012). Training followed the overload principle: 3 x 60s (1 $x$ 60s for each exercise, with 30s rest between each) at a frequency of $35 \mathrm{~Hz}$ and amplitude of $2 \mathrm{~mm}$ (acceleration $=\sim 9.86 \mathrm{~g})$ in week one; $3 \times 70 \mathrm{~s}$ at $35 \mathrm{~Hz} / 2 \mathrm{~mm}$ $(\sim 9.86 \mathrm{~g})$ in week two; $3 \times 60 \mathrm{~s}$ at $40 \mathrm{~Hz} / 2 \mathrm{~mm}(\sim 12.9 \mathrm{~g})$ in week three; $3 \times 70 \mathrm{~s}$ at $40 \mathrm{~Hz} / 2 \mathrm{~mm}(\sim 12.9 \mathrm{~g})$ in week four; $3 \times 60 \mathrm{~s}$ at $45 \mathrm{~Hz} / 2 \mathrm{~mm}(\sim 16.3 \mathrm{~g})$ in week five; and $3 \times 70 \mathrm{~s}$ at $45 \mathrm{~Hz} / 2 \mathrm{~mm}(\sim 16.3 \mathrm{~g})$ in week six. The protocol was selected to reflect previous research suggesting that frequencies between $35 \mathrm{~Hz}$ and $50 \mathrm{~Hz}$ can improve vertical jumping performance in untrained (Delecluse et al., 2003; Bazett-Jones, Finch, \& Dugan, 2008) and trained individuals (Hawkey et al., 2009a). The acceleration of vibration was calculated in accordance with Hawkey (2012) (Figure 2).

Prior to, and on completion of, the study all participants performed three maximal countermovement jumps (CMJ); reported to be an accurate and reliable method of assessing body size-independent muscular power (Markovic \& Jaric, 2007). All jumps were performed on a contact mat (Just Jump: Probotics Inc. USA); utilised in a number of previous studies (Christensen and Nordstrom, 2008; Delecluse et al., 2003; Hawkey et al., 2012) and shown to be a reliable method

FIGURE 2

Formula used to calculate acceleration of vibration (adapted from Hawkey, 2012)

$$
a=\frac{A \times(2 \pi f)^{2}}{g}
$$


of assessing jump performance (Isaacs, 1998), with high criterion validity (Leard et al., 2007). During the jump testing, hands were required to remain on the hips in accordance with previous research (Hawkey et al., 2012; Linthorne, 2001) in an attempt to standardise jumping technique and because arm use has a significant impact on jumping performance (Linthorne, 2001). Knee angles for the jumps were initially measured using a goniometer, and were then controlled visually to ensure consistency. All CMJ data was analysed using a 2-way ANOVA with repeated mea- sures, with one between-subject factor "group" (experimental vs. control) and one within-subject factor "time" (pre vs. post intervention).

\section{RESULTS}

A 2-way ANOVA with repeated measures indicated that there was a significant time $\mathrm{x}$ group interaction $(p<.0001)$ for CMJ performance between the intervention (pre- $.43 \pm .08 \mathrm{~m}$ to post- $.49 \mathrm{~m} \pm .08$ ) and the control group (pre- $.43 \pm .07 \mathrm{~m}$ to post- .41 $\pm .08 \mathrm{~m})$ (Figure 3).

\section{FIGURE 3}

Plot from the 2-way ANOVA showing pre- and post-jump performance in the vibration and control groups

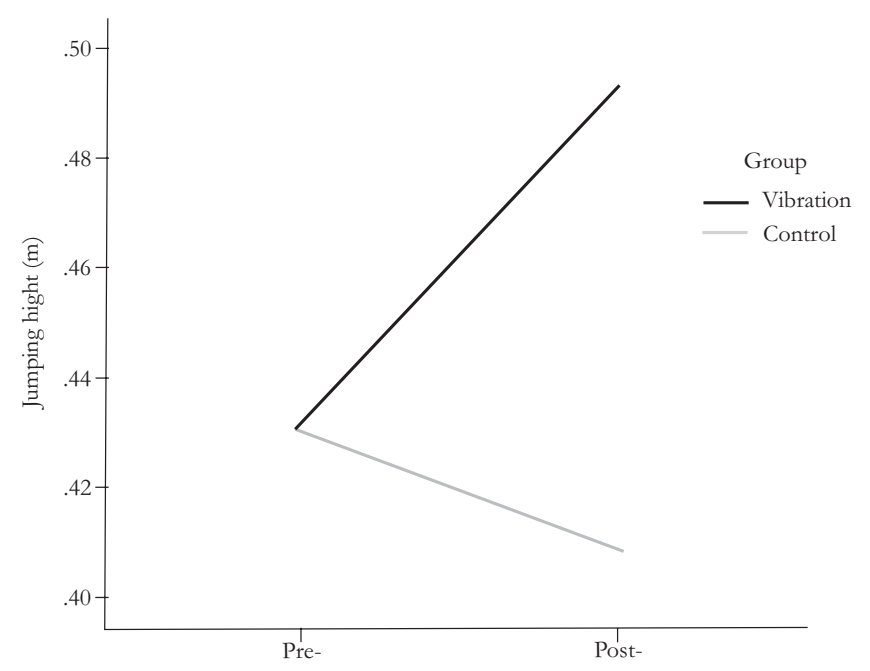

\section{DISCUSSION}

Previous research into the effects of WBV has shown improvements in a variety of performance measures in a range of populations. Studies conducted on footballers and athletes (Hawkey et al., 2009a, 2009b; Hawkey et al., 2012; Paradisis \& Zachardiogiorgis, 2007) and sedentary individuals (Delecluse et al., 2003; Torvinen et al., 2002) have all highlighted that WBV has the potential to improve activity specific performance. Results of the current study show that 6-weeks WBV training is sufficient to elicit significant improvements in vertical jumping performance in a recreationally active male population. The difference between the pre- and post- jumping performance of the WBV group equates to a $>14 \%$ improvement, while the control group experienced a $3 \%$ reduction in pre- to post- measures; resulting in a significant time $\mathrm{x}$ group interaction $(p<.0001)$. Despite the current study only being conducted over a period of 6-weeks the findings show similarities with the studies of Delecluse et al. (2003) and Torvinen et al. (2002), which were conducted over 12-we- ek and 4-month periods, respectively. However, it is difficult to directly compare these studies due to differences in experimental protocols, the different equipment used for both testing and training, and the variation of performance level and training experience of the participants. This is highlighted by Moras, Tous, Muñoz, Padullés, and Vallejo (2006) who state that differences in their results when compared against other similar studies could have been due to different equipment (NEMES versus PowerPlate), or different samples employed in the respective trials. One difference in protocol is that Delecluse et al. (2003) used only female participants, while the current study utilised a male only population. This is potentially significant because, according to Bazett-Jones et al. (2008), men and women respond differently to vibratory stimuli. There is also an obvious disparity in the frequency and amplitude of the platform utilised in the current study compared to some previous research. While it has been widely reported that a frequency of $30 \mathrm{~Hz}$ is conducive to improvements in muscular power (Paradisis \& Zachardiogiorgis, 2007), it is currently unclear why this is the case and there 
is currently confusion regarding how certain variables used in vibration training (frequency, amplitude and acceleration) are reported and quantified (Hawkey, 2012; Lorenzen, Maschette, Koh, \& Wilson). The justification for using a frequency range of $35-45 \mathrm{~Hz}$ and amplitude of $2 \mathrm{~mm}$ was in accordance with previous research (Delecluse et al., 2003; Hawkey et al., 2009a, 2009b; Hawkey et al., 2012), which had reported improvements in jumping performance within these ranges. The squatting exercises $\left(90^{\circ}-120^{\circ}\right)$ used for the current study were also used in accordance with previous research showing favourable outcomes in jumping performance (Hawkey et al., 2012; Paradisis \& Zachardiogiorgis, 2007).

It is also difficult to quantify the mechanisms involved in the reported performance improvements. Enhancements in muscular performance following WBV are similar to those seen after explosive power training (Bosco, Colli, \& Introini, 1999). As the first mechanism involved in skeletal muscle adaptation is neural (Delecluse et al., 2003), it is therefore likely that the improvements in performance, reported following WBV, are also likely to originate from neural adaptations. The mechanisms of these improvements are likely to be through stimulation of the primary endings of the muscle spindle, resulting in a tonic contraction of the muscle, known as the tonic vibration reflex (Mester et al., 2006), increases in motor unit synchronisation, co-contraction of the synergist muscles, and increased inhibition of the antagonist muscles (Hawkey, 2012). It is feasible to suggest that improvement in muscular power in the 12-week (Delecluse et al., 2003) and 4-month (Torvinen et al., 2002) studies were not only influenced by neural adaptations (as mentioned above), but also through intramuscular factors such as enlargement of slow- and fast-twitch fibres; as has been reported in studies using vibration exposed rats (Necking, Lundstrom, Lundborg, Thornell, \& Friden, 2006).

The results from this current study add to the growing evidence about the benefits of vibration training on a range of performance measures in a variety of populations. Combined with the findings of Delecluse et al. (2003) and Torvinen et al. (2002) in particular, this current study's findings are encouraging for those individuals, who are sedentary or inactive, wanting to improve their muscular strength following a relatively low impact, low intensity exercise intervention. However, there are some limitations with the current research. Although the study was very well controlled regarding the standardisation of training protocols, and performance level of the participants, there was no regulation of participants' activity outside of the confines of the study. It is therefore feasible that participants, in either the control or the WBV group, undertook additional training during the intervention; this could have contributed to the improved jumping performance of the WBV group or jeopardised the control group due to muscle fatigue. That the acceleration rate was not quantified or verified using accelerometers, as was the case during the Delecluse et al. (2003) study is also a negative aspect of the current research.

\section{CONCLUSION}

Results from the current study appear to suggest that a 6-week WBV training programme, conducted once-a-week, has the potential to significantly improve the jumping performance, and therefore muscular power, of a recreationally active male population. While results from this current and other previous studies would lend support to the inclusion of vibration interventions for a range of health, fitness, and performance improvements it is currently unclear as to the exact mechanisms influencing these benefits and the potential impact, if any, of any external training performed outside the realms, and control, of the current study. Future research should now focus on ascertaining the optimum frequencies and amplitudes conducive to maximising performance improvements. The effect of activity/performance level, the duration and specific exercises performed on the vibration platform, and also any age and sex differences would also be useful variables to investigate further.

\section{REFERENCES}

Bazett-Jones, D. M., Finch, H. W., \& Dugan, A. L. (2008). Comparing the effects of various wholebody vibration accelerations on countermovement jump performance. Journal of Sports Science and Medicine, 7, 144-150.

Bosco, C., Colli, R., \& Introini, E. (1999). Adaptive responses of human skeletal muscle to vibration exposure. Clinical Physiology, 19, 183-187. doi: 10.1046/j.1365-2281.1999.00155.x; PMid: 10200901

Bruyere, O., Wuidart, M. A., Di Palma, E., Gourlay, M., Ethgen, O., Richy, F., \& Reginster, J. Y. (2005). Controlled whole body vibration to decrease fall risk and improve health-related quality of life of nursing home residents. Archives of Physical Medicine and Rehabilitation, 86(2), 303-307. doi: 10.1016/j. apmr.2004.05.019; PMid: 15706558 
Cardinale, M., \& Bosco, C. (2003). The use of vibration as an exercise intervention. Exercise and Sport Science Reviews, 31, 3-7. doi: 10.1097/ 00003677-200301000-00002

Delecluse, C., Roelants, M., \& Verschueren, S. (2003). Strength increase after whole-body vibration compared with resistance training. Medicine and Science in Sport and Exercise, 35, 1003-1041. doi: 10.1249/01.MSS.0000069752. 96438.B0; PMid: 12783053

Eklund, G., \& Hagbarth, K. E. (1966). Normal variability of tonic vibration reflex. Experimental Neurology, 16, 80-92. doi: 10.1016/00144886(66)90088-4

Fischbach, A. (2007). Shaky ground. Fitness Business, Pro, October Issue.

Hawkey, A., Morrison, D., Williams, D., \& Nevill, A. (2009a). Effect of a five-week whole body vibration training programme on vertical jump performance in male professional football goalkeepers. Journal of Sports Sciences, 27(4), S138.

Hawkey, A., Lau, Y., \& Nevill, A. (2009b). Effect of six-week whole body vibration training on vertical jump and flexibility performance in male national league basketball players. Journal of Sport Sciences, 27(4), S138-S139.

Hawkey, A. (2012). Editorial: Quantification, clarification and standardisation of whole body vibration. Journal of Sports Therapy, 5(1), Editorial paper n. p.

Hawkey, A., Evans, R., \& Nevill, A. (2012). Whole body vibration training improves sport specific performance measures in semi-professional footballers. Proceedings of British Association of Sport and Exercise Sciences (BASES) Biomechanics Interest Group Annual Meeting, 27: 30. Retrived from http://www.science.ulster.ac.uk/big2012/ public/pdf/BIG_2012_Proceedings_Ulster.pdf

Hong, J., Kipp, K., Maddalozzo, G., \& Hoffman, M. A. (2010). Acute effects of whole body vibration on rate of force development and electromechanical delay. Journal of Sports Therapy, 3(3), 3-9.

Issurin, V. B., \& Tenenbaum, G. (1999). Acute and residual effects of vibratory stimulation on explosive strength in elite and amateur athletes. Journal of Sports Sciences, 17, 177-82. doi: 10.1080/026404199366073; PMid: 10362384

Kumari, R., Wyon, M., Hawkey, A., \& Metsios, G. (2011). Effects of vibration on disease activity scores in patients with rheumatoid arthritis: a case study. Journal of Sports Therapy, 4(1): 30-33.

Leard, J. S., Cirillo, M. A., Katsnelson, E., Kimiatek, D. A., Miller, T. W., Trebincevic, K., \&
Garbalosa, J. C. (2007). Validity of two alternative systems for measuring vertical jump height. Journal of Strength and Conditioning Research, 21(4): 1296-1299. doi: 10.1519/00124278200711000-00055; doi: 10.1519/R-21536.1; PMid: 18076265

Liebermann, D. G., \& Issurin, V. B. (1997). Effort perception during isotonic muscle contractions with superimposed mechanical vibration stimulation. Journal of Human Movements Studies, 32, 171-186.

Linthorne, N. P. (2001). Analysis of standing vertical jumps using a force platform. American Journal of Physics, 69(11), 1198-1204. doi: 10.1119/1.1397460

Lorenzen, C., Maschette, W., Koh, M., \& Wilson, C. (2009). Inconsistent use of terminology in whole body vibration exercise research. Journal of Science and Medicine in Sport, 12, 676-678. doi: 10.1016/j.jsams.2008.06.008; PMid: 18762453

Luo, J., McNamara, B., \& Moran, K. (2005). The use of vibration training to enhance muscle strength and power. Sports Medicine, 35, 23-41. doi: 10.2165/00007256-200535010-00003; PMid: 15651911

Markovic, G., \& Jaric, S. (2007). Is vertical jump height a body size-independent measure of muscle power? Journal of Sports Sciences, 25(12), 1355-1363. doi: 10.1080/02640410601021713; PMid: 17786688

Mester, J., Kleinoder, H., \& Yue, Z. (2006). Vibration training: benefits and risks. Journal of Biomechanics, 39(6), 1056-1065. doi: 10.1016/j. jbiomech.2005.02.015; PMid: 15869759

Moras, G., Tous, J., Muñoz, C. J., Padullés, J. M., \& Vallejo, L. (2006). Electromyographic response during whole-body vibrations of different frequencies with progressive external loads 10(93). Retrived from http://www.efdeportes. com/efd93/emg.htm.

Necking, L. E., Lundstrom, R., Lundborg, G., Thornell, L. E., \& Friden, J. (1996). Skeletal muscle changes after short term vibration. Scandinavian Journal of Plastic and Reconstructive Surgery and Hand Surgery, 30, 99-103. doi: 10.3109/02844319609056390; PMid: 8815978

Nordlund, M. M., \& Thorstensson, A. (2007) Strength training effects of whole-body vibration? Scandinavian Journal of Medicine and Science in Sports, 17, 12-17.

Paradisis, G., \& Zacharogiannis, E. (2007). Effects of whole-body vibration training on sprint running kinematics and explosive strength 
performance. Journal of Sports Science and Medicine, 6, 44-49.

Riley, W., \& Sturges, L. (1996). Engineering Mechanics: Dynamics. 2nd ed. New York, NY: John Wiley \& Sons, Inc.

Ronnestad, B. R. (2004). Comparing the performance-enhancing effects of squats on a vibration platform with conventional squats in recreationally resistance-trained men. Journal of Strength and Conditioning Research, 18(4), 839-845. doi: 10.1519/00124278-200411000-00027; doi: 10.1519/14573.1; PMid: 15574092
Rubin, C., Recker, R., Cullen, D., Ryaby, J., McCabe, J., \& McLeod, K. (2004). Prevention of postmenopausal bone loss by a low-magnitude, high-frequency mechanical stimuli: a clinical trial assessing compliance, efficacy and safety. Journal of Bone and Mineral Research, 19(3), 343-351. doi: 10.1359/JBMR.0301251; PMid: 15040821

Torvinen, S., Kannu, P., \& Sievanen, H. (2002). Effect of four-month vertical whole body vibration on performance and balance. Medicine and Science in Sport and Exercise, 34, 1523-1528. doi: 10.1097/00005768-200209000-00020

Received: May 11, 2012

Revision received: December 1, 2012

Accepted: December 7, 2012

Correspodence to:

Adam Hawkey, BSc(Hons) MSc FBIS

Research Centre for Sport, Exercise and Performance

University of Wolverhampton

Gorway Road

Walsall

WS1 3BD

United Kingdoms

Phone: 00441902322824

Fax: 00441902323295

E-mail: A.Hawkey@wlv.ac.uk 